\title{
Osmotic Poisson's Ratio and Equilibrium Stress of Poly(acrylamide) Gels
}

\author{
Toshikazu Takigawa, ${ }^{\dagger}$ Yoshiro Morino, Kenji Urayama, ${ }^{*}$ and Toshiro Masuda \\ Department of Material Chemistry, Kyoto University, Sakyo-ku, Kyoto 606-01, Japan \\ *Institute for Chemical Research, Kyoto University, Uji, Kyoto 611, Japan
}

(Received May 31, 1996)

KEY WORDS Polymer Gel / Swelling under Elongation / Osmotic Poisson's Ratio / Equilibrium Stress /

Polymer gels have been recognized as a unique and interesting soft material, and the physical and mechanical properties of polymer gels have been investigated by many researchers. ${ }^{1-3}$ Experimental and theoretical studies, especially, on the volume phase transition ${ }^{1}$ and swelling kinetics, ${ }^{1,2}$ have been carried out intensively. The physical properties of polymer gels have become clear. We have already shown ${ }^{4}$ that the swelling kinetics of polymer gels can be written by two types of diffusion equations, i.e., the equations of longitudinal and transverse modes. The former determines the time evolution of the volume of gels from the initial to final state, and the latter the time dependence of the change of shape. We also found that the uniaxially stretched gel in solvent swells gradually until equilibrium under the deformation is attained. ${ }^{4}$ When a gel sample is deformed quickly in solvent, the state just after the application of the strain, which we call hereafter the initial state, must be determined by the material nature of the gel used. For example, in the case that a gel specimen is stretched uniaxially, the initial dimension of the specimen perpendicular to the elongation is determined by Poisson's ratio as a material constant $\left(\mu_{0}\right)$. This holds so long as the time for swelling is long enough compared with the time required for the mechanical measurement to estimate Poisson's ratio, and this situation is easily attained by employing a larger size of polymer gel.

On the other hand, the final state, i.e., the state realized at long time limit after the application of the deformation, will be determined not only by the mechanical properties of the gel (for example, $\mu_{\infty}$ of the gels), but also by thermodynamic properties of the system under deformation. The polymer gel and surrounding solvent molecules compose a semi-open system and the solvent molecules can move through the interface between gel and solvent during long time when the solvent motion is favored. Osmotic Poisson ratio $\left(\mu_{\infty}\right)$ defined for uniaxially deformed gels in solvent at long time limit reflects the thermodynamic properties of the semi-open system.

The equilibrium elongational stress $\left(\sigma_{\infty}\right)$ has also been affected by the thermodynamic properties of the system. Using the initial elongational stress $\left(\sigma_{0}\right)$ and $\sigma_{\infty}$, we can define the relative stress reduction $\left(\sigma_{0}-\sigma_{\infty}\right) / \sigma_{0}$. This quantity is also affected by the thermodynamic properties of the gel system. Although $\mu_{\infty}$, and $\sigma_{\infty}$ or $\left(\sigma_{0}-\sigma_{\infty}\right) / \sigma_{0}$ are fundamental quantities for describing

\footnotetext{
† To whom correspondence should be addressed.
}

the physical and mechanical properties of polymer gels at equilibrium, there are few studies on those values. ${ }^{4-7}$ The aim of the present study is to investigate the values of $\mu_{\infty}$ and $\left(\sigma_{0}-\sigma_{\infty}\right) / \sigma_{0}$ for poly(acrylamide) (PAAm) gels, and to compare those with the theoretical predictions shown in a previous paper. ${ }^{4}$

\section{EXPERIMENTAL}

PAAm gels used in this study were prepared by employing a radical polymerization technique. Acrylamide (AAm), methylenebisacrylamide (MBA; crosslinker), and ammoniumpersulfate (initiator) were dissolved in distilled water at room temperature. The molar ratio of MBA to AAm and that of initiator to AAm were 0.002 and 0.006 , respectively. The solution was poured in a metal mold in order to obtain a rectangular specimen. The gelation was performed at $33^{\circ} \mathrm{C}$. After being removed from the mold, the gel specimen was immersed in distilled water until the equilibrium swelling was achieved. The sample name in Table I contains three digits. The left two digits represent the initial concentration of AAm in $10 \mathrm{~kg} \mathrm{~m}^{-3}$, and the rest the serial number. The cross-sectional area of the rectangular sample, after equilibrium swelling, was square in shape and was about $3 \mathrm{~mm} \times 3 \mathrm{~mm}$ for PAAm 277 and PAAm $278, c a .6 \mathrm{~mm} \times 6$ $\mathrm{mm}$ for PAAm 275 and PAAm 276, and $c a .9 \mathrm{~mm} \times 9 \mathrm{~mm}$ for the other samples. The gel specimens were elongated in water at $25^{\circ} \mathrm{C}$ by using an Orientec RTM-250 tensile tester with a specially designed water bath, and time dependence of the tension was monitored. Two types of strain were defined for the stretched direction; one is the global strain and was calculated by using the crosshead distance, and the other is the local strain and was calculated from the distance between two marked points at the center of the gel sample by using a magnified video picture on the monitor screen. On the other hand, only the local strain, which was determined by the dimensional change of the gel in the central region, was employed for the direction perpendicular to the elongation. The global strain initially applied to the gel samples was fixed to be $10 \%$. The local strains were used for calculation of $\mu_{\infty}$, while the global strains were used for calculation of the initial Young's modulus $\left(E_{0}\right)$.

\section{RESULTS}

The experimental results on the values of $\mu_{\infty}$ and $\left(\sigma_{0}-\sigma_{\infty}\right) / \sigma_{0}$ for the PAAm gels are summarized in Table 
Table I. Sample name, initial Young's modulus $\left(E_{0}\right)$, osmotic Poisson's ratio $\left(\mu_{\infty}\right)$, and relative stress reduction $\left(\left(\sigma_{0}-\sigma_{\infty}\right) / \sigma_{0}\right)$ for poly(acrylamide) (PAAm) gels

\begin{tabular}{cccc}
\hline Sample & $E_{0} / \mathrm{k} \mathrm{Pa}^{\mathrm{a}}$ & $\mu_{\infty}$ & $\left(\sigma_{0}-\sigma_{\infty}\right) / \sigma_{0}$ \\
\hline PAAm271 & 22.9 & - & 0.271 \\
PAAm272 & 41.0 & 0.130 & 0.164 \\
PAAm273 & 38.2 & 0.116 & 0.173 \\
PAAm274 & 35.0 & 0.128 & 0.282 \\
PAAm275 & 49.5 & 0.141 & 0.215 \\
PAAm276 & 30.0 & 0.202 & 0.199 \\
PAAm277 & 43.0 & - & 0.194 \\
PAAm278 & 31.0 & - & 0.138 \\
PAAm191 & 9.6 & - & 0.242 \\
PAAm201 & 12.5 & 0.136 & 0.149 \\
PAAm331 & 78.0 & 0.155 & 0.216 \\
PAAm401 & 100 & 0.187 & 0.146 \\
\hline
\end{tabular}

${ }^{\text {a }}$ Calculated by using global strain.

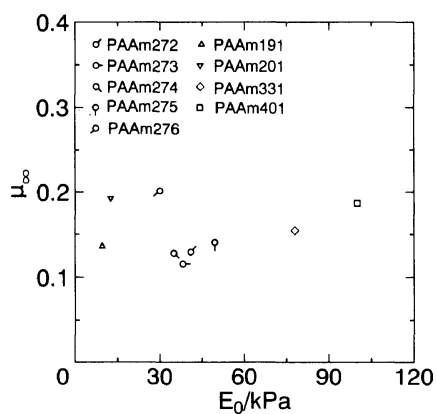

Figure 1. Osmotic Poisson's ratio $\left(\mu_{\infty}\right)$ of poly(acrylamide) (PAAm) gels plotted against initial Young's modulus $\left(E_{0}\right)$.

I. The values of $E_{0}$ for PAAm 271 to PAAm 278 were a little scattered, although we tried to prepare the gels with almost the same $E_{0}$ by fixing the concentration of the reagents as well as the conditions for gelation. Comparing the $E_{0}$ values for the specimens differing in the AAm concentration, $E_{0}$ becomes higher with increasing AAm concentration. The plot of $\mu_{\infty}$ against $E_{0}$ is shown in Figure 1. The data points are a little scattered but they appear to be independent of the AAm concentration. The average value with the standard deviation is $0.149 \pm 0.030$. Figure 2 shows the plot of $\left(\sigma_{0}-\sigma_{\infty}\right) / \sigma_{0}$ against $E_{0}$. The data points are also independent of $E_{0}$ in this region of $E_{0}$. The average value of $\left(\sigma_{0}-\sigma_{\infty}\right) / \sigma_{0}$ is 0.199 with the standard deviation 0.048 .

\section{DISCUSSION}

First, we compare the experimental values of $\mu_{\infty}$ and $\left(\sigma_{0}-\sigma_{\infty}\right) / \sigma_{0}$ with theoretical ones shown in a previous paper, ${ }^{4}$ where an isotropic gel with low polymer concentration stretched uniaxially in solvent was considered. The gel was also assumed to be incompressible. Starting from the Flory-type Gibbs free energy per unit volume, ${ }^{4,8}$ the osmotic stresses parallel and perpendicular to the stretched direction $\left(\Pi_{\| /}\right.$and $\Pi_{\perp}$, respectively) are given by $^{4}$

$$
\begin{aligned}
& \Pi_{/ /}=(5 / 2) G \varepsilon_{/ /}+G \varepsilon_{\perp}-\sigma_{\infty} \\
& \Pi_{\perp}=(1 / 2) G \varepsilon_{/ /}+3 G \varepsilon_{\perp}
\end{aligned}
$$

Here, $G$ is the shear modulus. The quantities, $\varepsilon_{/ /}$and $\varepsilon_{\perp}$

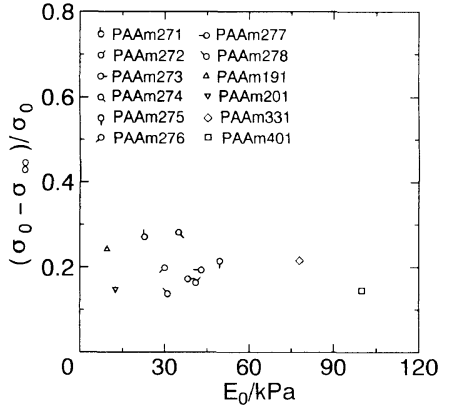

Figure 2. Relative stress reduction $\left(\left(\sigma_{0}-\sigma_{\infty}\right) / \sigma_{0}\right)$ of poly(acrylamide) (PAAm) gels plotîed against initial Young's modulus $\left(E_{0}\right)$.

are the strains parallel and perpendicular to the stretched direction at equilibrium. The above equations hold only for small $\varepsilon_{\| /}$. At equilibrium, $\Pi_{\| /}$and $\Pi_{\perp}$ are zero. It is clear from eq 2 that $\mu_{\infty}=1 / 6,{ }^{4}$ since $\mu_{\infty}$ is defined by $\mu_{\infty}=-\varepsilon_{\perp} / \varepsilon_{\|}$. The average value of $\mu_{\infty}$ for PAAm gels experimentally obtained in this study $(=0.149)$ coincides fairly well with the theoretical prediction $\left(\mu_{\infty}=1 / 6 \cong\right.$ $0.167)$. Geissler et al. ${ }^{5-7}$ have reported $\mu_{\infty}=0.275 \pm$ 0.011 for PAAm gel in their latest work. ${ }^{5}$ Their value is high compared with that obtained in this study. Geissler et al. have estimated $\mu_{\infty}$ from the combination of two different moduli, while we measured the value directly from the dimensional change of the sample in solvent under uniaxial deformation. From eq 1 and 2, we have $\sigma_{\infty}=(7 / 3) G \varepsilon_{/ /}$. The initial stress $\left(\sigma_{0}\right)$ is given by $3 G \varepsilon_{/}$ when the gel is incompressible as assumed in the theory. ${ }^{4}$ In this case, $\left(\sigma_{0}-\sigma_{\infty}\right) / \sigma_{0}$ results theoretically in the value of $2 / 9 \cong 0.222$. The average value of $\left(\sigma_{0}-\sigma_{\infty}\right) / \sigma_{0}$ for the PAAm gels investigated here was 0.199 , as shown previously. The difference in $\left(\sigma_{0}-\sigma_{\infty}\right) / \sigma_{0}$ between experiment and theory is not so large. By using the value of $\left(\sigma_{0}-\sigma_{\infty}\right) / \sigma_{0}$, we can also estimate $\mu_{\infty}$ for the PAAm gels by experiment when $\mu_{0}$ is known, since $\sigma_{0}=$ $\left(1+\mu_{0}\right) G \varepsilon_{/ /}$and $\sigma_{\infty}=\left(1+\mu_{\infty}\right) \varepsilon_{/ /}$. For the PAAm gels examined here, we have $\left(\mu_{0}-\mu_{\infty}\right) /\left(1+\mu_{0}\right)=0.199$. The PAAm gels were not actually incompressible and $\mu_{0}$ of the gels ${ }^{9}$ was about 0.46 , which gives $\mu_{0} \cong 0.17$. This is close to the value $\left(\mu_{\infty}=0.149\right)$ obtained directly by the ratio $\left(-\varepsilon_{\perp} / \varepsilon_{\|}\right)$, indicating that the two values of $\mu_{\infty}$ obtained independently by experiment is consistent with each other.

\section{REFERENCES}

1. See, for example, articles published in Adv. Polym. Sci., 109 and 110 (1993).

2. A. Onuki and K. Kawasaki, Ed., "Dynamics and Patterns in Complex Fluids," Springer, Berlin and Heidelberg, 1990.

3. W. Burchard and S. B. Ross-Murphy, Ed., "Physical Networks. Polymers and Gels," Elsevier Applied Science, London and New York N.Y., 1990.

4. T. Takigawa, K. Urayama, Y. Morino, and T. Masuda, Polym. J., 25, 929 (1993)

5. E. Geissler, A. M. Hecht, F. Horkay, and M. Zrinyi, Macromolecules, 21, 2594 (1988).

6. E. Geissler and A. M. Hecht, Macromolecules, 13, 1276 (1980).

7. E. Geissler and A. M. Hecht, Macromolecules, 14, 185 (1981).

8. S. Hirotsu and A. Onuki, J. Phys. Soc. Jpn., 87, 1392 (1987).

9. T. Takigawa, Y. Morino, K. Urayama, and T. Masuda, Polym. Gels Networks, 4, 1 (1996). 